\title{
Multi-epoch VLBI Images to Study the ICRF-3 Defining Sources in the Southern Hemisphere
}

\author{
Sayan Basu*, Aletha de Witt, Jonathan Quick \\ Hartebeesthoek Radio Astronomy Observatory \\ (A Research Facility under the South African Radio Astronomical Observatory) \\ P.O Box 443, Krugersdorp 1740 \\ South Africa \\ E-mail: sayan@hartrao.ac.za
}

\section{Zinovy Malkin}

Pulkovo Observatory

St. Petersburg, Russia

\begin{abstract}
The third realisation of the International Celestial Reference Frame (ICRF-3) is based on high precision Very Long Baseline Interferometric (VLBI) measurements of positions of 4,588 extragalactic radio sources and 4,536 sources of them are observed at 2.3 and $8.4 \mathrm{GHz}$. This includes the 303 defining sources which determine the orientation of the frame axes. These reference sources often show extended emissions on milliarcsecond scales that can vary with time, frequency and baseline projection. The effect of source structure on astrometric VLBI positions can be significant and it is therefore important to map the structures of these sources on a regular basis. However, due to the limited number of radio telescopes in the south, it has always been difficult to run dedicated imaging programmes and to monitor source structure, especially for sources below $-30^{\circ}$ declination. We present results from our efforts to image and monitor the intrinsic source structures of ICRF sources using ongoing astrometric and geodetic VLBI observations in the south.
\end{abstract}

14th European VLBI Network Symposium Users Meeting (EVN 2018)

8-11 October 2018

Granada, Spain

${ }^{*}$ Speaker. 


\section{Introduction}

The International Celestial Reference Frame (ICRF; [1]) is based on high precision Very Long Baseline Interferometric (VLBI) measurements of positions of extragalactic radio sources at 2.3 (S-band) and $8.4 \mathrm{GHz}$ (X-band). In turn, VLBI observations of these extragalactic sources are crucial to many applications, for example the realization of the International Terrestrial Reference Frame (ITRF), calculating the orientation of the Earth in space, providing calibrator sources for parallax measurements, for determining high accuracy positions of other radio sources, as well as for imaging faint sources in astronomy, studying the motion of tectonic plates, measurements of the sea-level rise and spacecraft navigation, to name but a few. The present realization of the ICRF, the ICRF-3 [2] contains accurate positions of 4,588 radio sources, of which 4,536 radio sources are observed at $\mathrm{S} / \mathrm{X}$-band. Among these 4,536 radio sources 303 radio source positions are used to determine the orientation and stability of the ICRF axes. These 303 sources are known as the defining sources.

It is a well known fact that radio reference frames are weaker in the south. The smaller number of radio telescopes and thus observing sessions in the south have always resulted in catalogs of radio sources being much weaker in the south (especially below $-30^{\circ}$ declination), in both density and precision. Although the ICRF-3 provided a major improvement in the density and precision of the ICRF in the south, there still remains a significant hemisphere disparity in source distribution and density. In Figure 1, we show the sky distribution of ICRF-3 sources using a method of subdividing a spherical surface into 406 equal-area cells [3]. It is clear that we still need more sources in the south and that we need to improve the spatial coverage, especially for declinations south of $-30^{\circ}$ declination.

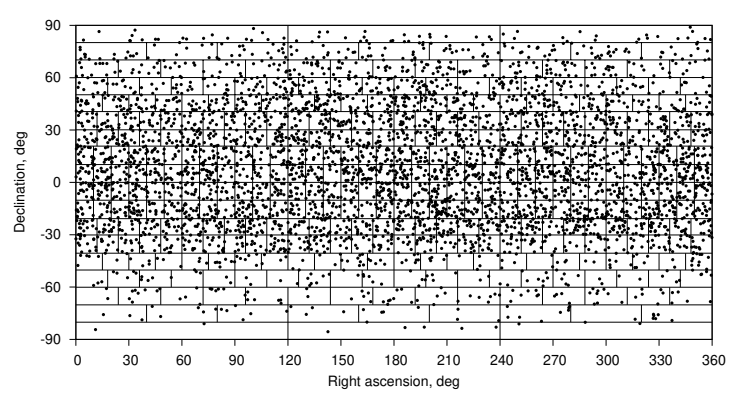

(a)

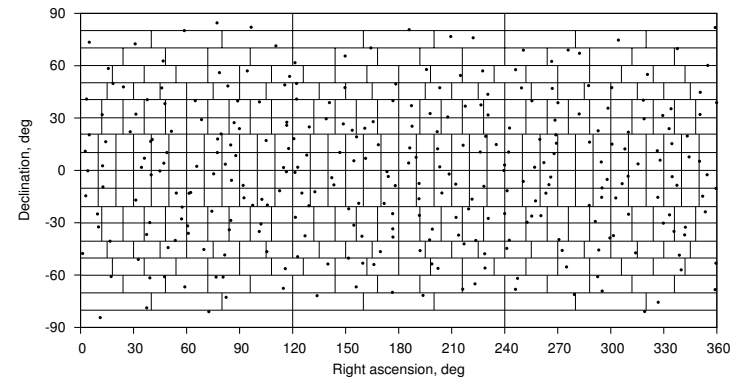

(b)

Figure 1: Sky distribution of (a) ICRF-3 sources and (b) ICRF-3 defining sources only. The entire sky was divided into 406 equal-area cells where each cell has an area of $\sim 101$ sq. degree.

The ideal candidates for celestial reference frames are those extragalactic radio sources that appear to have a point-like structure. However, in practice many sources exhibit extended structures on milliarcsecond scales that depend on frequency band and can vary with time. These extended structures can have serious effects on geodetic and astrometric VLBI measurements and multiepoch images are essential to assess the astrometric suitability of these reference frame sources. However, dedicated multi-epoch VLBI campaigns to map and monitor source structure in the south have proven difficult to obtain. We identified 124 sources ( 37 of which are ICRF-2 defining 
sources) in the far-south (below $-45^{\circ}$ declination), with no VLBI images and only 12 sources with images at more than 10 epochs. Multi-epoch VLBI images of these southern sources are needed to provide routine analysis of their astrometric quality.

\section{Observations and Results}

An astrometric VLBI programme named the Celestial Reference frame Deep South (CRDS) was selected to study source structure effects of reference frame sources in the south. There are 5-6 CRDS sessions per year with 4-6 participating antennas in every session. We have shown that these CRDS sessions can be used to successfully image the intrinsic structures of ICRF sources in the south [4]. Since January 2018, we increased the data rate of the CRDS sessions from $256 \mathrm{Mbps}$ to $1 \mathrm{Gbps}$ and we also optimized the scheduling for both geodesy and imaging. The higher data rates and improved scheduling resulted in more sources per session and improved uv-coverage for imaging. Prior to release of the ICRF-3, potential defining sources were also added to the CRDS sessions to analyze their astrometric quality.

To date the CRDS-63, 66 and 68 sessions from 2013 and the CRDS-94 session from 2018 have been used to produce maps of ICRF sources in the south. A total 102 sources have been observed in these sessions of which 76 sources were ICRF-2 defining sources. Some ICRF sources were imaged for the first time using data from CRDS sessions. These images were used in selecting defining sources for the ICRF-3. Data were recorded in right circular polarization (RCP) with 6 IFs at S-band and 8 IFs at X-band. The Astronomical Image Processing System (AIPS; [5]) was used for data calibration and imaging was done in the Difmap [6]. In Figure 2 we show multiepoch maps at X-band, for a representative sample of our sources imaged using CRDS data. We also calculated the structure index or magnitude of the source structure [7], which is widely used to analyze the astrometric quality of radio sources. In Figure 3, we plot the structure index for a representative sample of sources that were observed in both the CRDS-66 and CRDS-68 sessions. A structure index of 1 represents a very good reference source while a 2 represents a good source, a 3 represents a source that should be used with caution and a 4 represents a source that is not recommend for use as a reference frame source.

\section{Acknowledgements}

This research is supported by the Hartebeesthoek Radio Astronomy Observatory, a facility of the National Research Foundation (NRF) of South Africa.

\section{References}

[1] Ma C., et al., 1998, AJ, 116, 516

[2] Chalot P., 2017, in 23rd EVGA Working Meeting, 58

[3] Malkin Z., 2016, ArXiv e-prints

[4] Basu S. et al., 2016, in IVS General Meeting Proceedings, 312

[5] Greisen, E. W., 1998, in Astronomical Data Analysis Software and Systems VIII, 204 


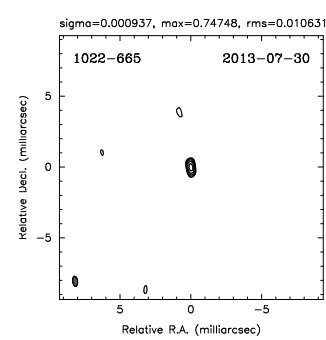

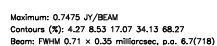

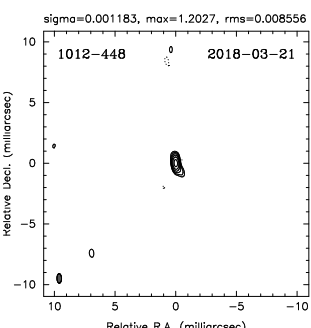

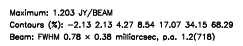

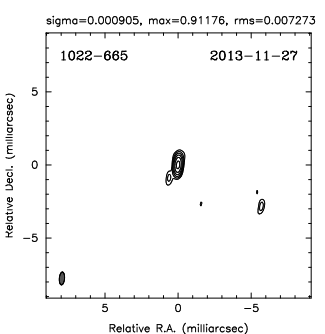

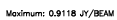
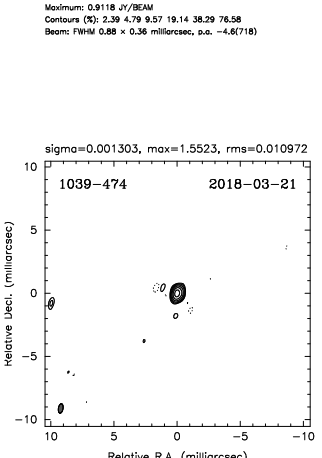

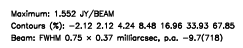

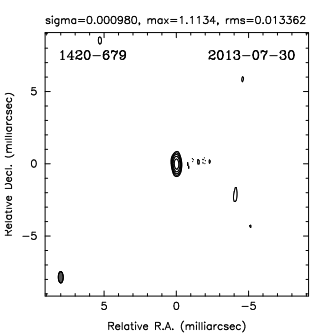

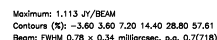

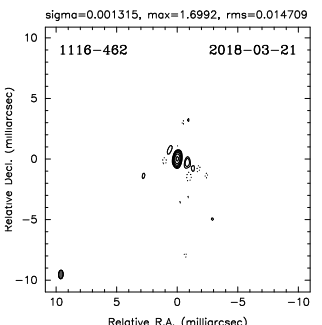

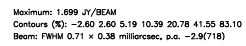

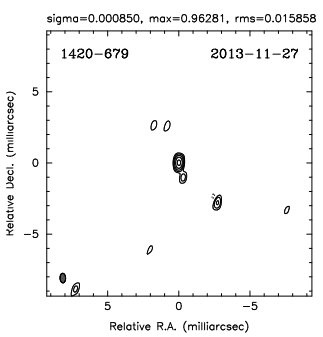

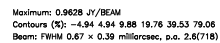

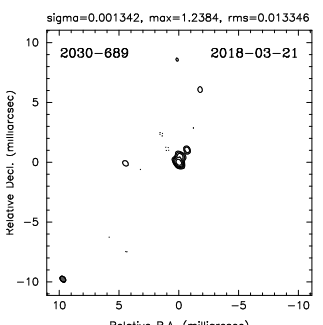

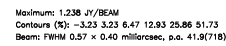

Figure 2: First row: Multi-epoch maps for two of the sources that was observed in both the CRDS66 and CRDS-68 sessions. Second Row: Maps of a representative sample of the sources that were observed in the CRDS-94 session. North is up and East is to the left.

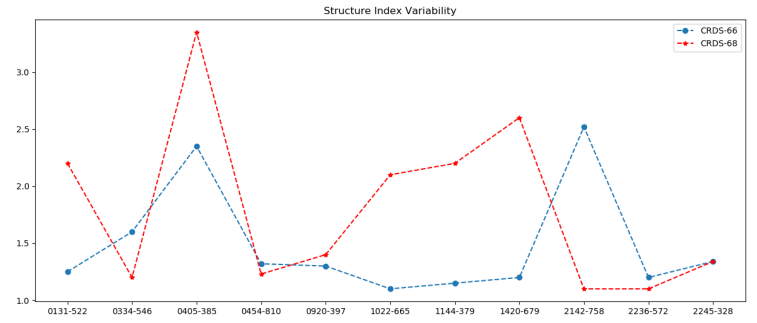

Figure 3: Structure index variability for a selection of sources that were observed in both the CRDS-66 and 68 session. The time gap between the two sessions are $\sim 6$ months. Blue circles and red stars represent the structure index calculated from CRDS-66 and in CRDS-68 respectively.

[6] Shepherd, M. C., Pearson, T. J. and Taylor, G. B., 1994, in Bulletin of the American Astronomical Society, 987

[7] Charlot P., 1990, AJ, 99, 1309-1326 